\title{
THE ABUNDANCE OF ATOMIC CARBON NEAR THE IONIZATION FRONTS IN M17 AND
} S140

\author{
Jocelyn Keene, Geoffrey A. Blake, and T. G. Phillips \\ California Institute of Technology \\ P. J. HugGins \\ New York University \\ AND \\ C. A. BEICHMAN \\ Jet Propulsion Laboratory, California Institute of Technology \\ Received 1984 December 10; accepted 1985 June 17
}

\begin{abstract}
We have observed the $492 \mathrm{GHz}$ ground-state line of atomic carbon in the edge-on ionization fronts in M17 and S140. We find that, contrary to expectation, the $\mathrm{C}$ I emission peaks farther into the molecular cloud from the ionization front than does the CO. In fact the peak C I abundance in M17 occurs more than 60 mag of visual extinction into the cloud from the ionization front. Calculations of the ratio of $\mathrm{C} I$ to $\mathrm{CO}$ column densities yield values of $0.1-0.2$. These observations do not support chemical models which predict that neutral atomic carbon should be found only near the edges of molecular clouds. Other models are discussed which may explain the observations.
\end{abstract}

Subject headings: interstellar: abundances — interstellar: matter — nebulae: abundances — nebulae: individual

\section{INTRODUCTION}

The initial study of the abundance of neutral atomic carbon (C I) in dense interstellar clouds by Phillips and Huggins (1981) showed that the ratio of $\mathrm{C}$ I to $\mathrm{CO}$ is surprisingly large. Early steady state ion-molecule chemistry calculations (e.g., Langer 1976a; Clavel, Viala, and Bel 1978; de Jong, Dalgarno, and Boland 1980) made the interesting prediction that the outer edges of molecular clouds would contain a considerable abundance of atomic carbon due to irradiation by the ambient interstellar UV field. However, these schemes in general also predicted that in the depths of dark clouds which have reached chemical equilibrium ( $>10^{6} \mathrm{yr}$ old) $\mathrm{C}_{\mathrm{I}}$ is less abundant than $\mathrm{CO}$ by several orders of magnitude. In contrast, column density ratios observed by Phillips and Huggins ranged from 0.04 to 0.7 , too large to be explained by the calculated cloud core values. The absolute column densities of $\mathrm{C} I$ are also too large to be explained by the edge contribution alone.

Since that time many physical and chemical models have been proposed to explain these large values. We have therefore embarked on a program of further observations, one set of which is described in this paper, to try to determine which of these models may be correct. Section II of this paper is a review of recent developments in chemical or physical models affecting carbon chemistry; Sections III and IV are descriptions of the objects we have observed (M17 and S140) and our observational procedures; $\S \S \mathrm{V}$ and VI contain the results, discussion, and summary.

\section{REVIEW OF RECENT CARBON CHEMISTRY MODELS}

The more recent chemical models which have tried to explain the observed large abundances of $\mathrm{C}_{\mathrm{I}}$ fall into two broad categories. The first type, which has several subclasses, produces $\mathrm{C}_{\mathrm{I}}$ in the bulk of clouds, while the second type produces $\mathrm{C}$ I only in radiation-dominated regions on the rims of clouds. Since there has been no recent review of the various models, we summarize them here.

\section{a) Time-dependent Chemistry}

Calculations of time-dependent chemical evolution show that the conversion of $\mathrm{C}$ I to $\mathrm{CO}$ should be nearly complete in $\sim 10^{6}$ yr (Langer 1976b; Iglesias 1977; Prasad and Huntress 1980; Graedel, Langer, and Frerking 1982; Leung, Herbst, and Heubner 1984). However the calculations of Graedel, Langer, and Frerking (1982) demonstrated that the time scale for conversion of $\mathrm{C} \mathrm{I}_{\mathrm{I}}$ to $\mathrm{CO}$ could be very long if the abundance of $\mathrm{H}_{3}{ }^{+}$(the primary species involved in the destruction of $\mathrm{C} \mathrm{I}$ ) is low. The abundance of $\mathrm{H}_{3}{ }^{+}$in turn was thought to be determined by the free electron abundance. A high electron abundance would suppress the $\mathrm{H}_{3}{ }^{+}$abundance, allowing $\mathrm{C}_{\mathrm{I}}$ to survive. Their results imply that in clouds with little depletion of metals there may be regions with large electron abundances which also have large residual abundances of $\mathrm{C} \mathrm{I}$, but there should be other regions, such as the cores of dark clouds, where the electon abundance is low and the abundance of $\mathrm{C} I$ is also low.

Unfortunately, the validity of these calculations can be questioned because of their use of a possibly incorrect rate coefficient for the dissociative electronic recombination of $\mathrm{H}_{3}{ }^{+}$. Smith and Adams (1984) measured a rate which is at least a factor of 100 smaller than assumed in these calculations. The effects of this are not yet completely known, but it seems that $\mathrm{H}_{3}{ }^{+}$might be more abundant than previously calculated. Thus the ion-molecule chemical reactions should be driven to completion everywhere, even where the electron abundance is high, leaving only a small residual of $\mathrm{C} \mathrm{I}$. Moreover, the calculations of Leung, Herbst, and Heubner (1984) for the large metal abundance case do not agree with those of Graedel, Langer, and

\section{(C) American Astronomical Society - Provided by the NASA Astrophysics Data System}


Frerking; instead, they show a very low equilibrium $\mathrm{C}$ I abundance at an age of $10^{7} \mathrm{yr}$ for all metal abundances at densities greater than $10^{4} \mathrm{~cm}^{-3}$.

\section{b) Dynamical Cloud Evolution}

An additional factor to consider in the evolution of clouds, besides time dependent chemistry, is dynamical evolution. Gerola and Glassgold (1978) and Tarafdar et al. (1985) have solved the chemical and dynamical problem for a set of clouds ranging in mass from 40 to $3 \times 10^{4} M_{\odot}$. They showed that (3-4) $\times 10^{6} \mathrm{yr}$ after clouds start collapsing, much of the carbon is still found in the form of $\mathrm{C}$. This is because the formation of CO takes place slowly at low densities, and the collapsing clouds spend much of their lifetime in a relatively diffuse state. Taking a cloud (3-4) $\times 10^{6} \mathrm{yr}$ old, Tarafdar et al. compared their predictions of the column density of $\mathrm{C}$ I versus visual extinction with previous observations of $\mathrm{C}$ I and found good agreement. Our more recent observations (this paper; also Frerking et al. 1985) are also in good agreement with this model. Unfortunately, the calculations were not carried past $4 \times 10^{6} \mathrm{yr}$, and it is commonly thought that many molecular clouds may be older than that. This model thus leaves us with the problem of delaying the chemical maturation of molecular clouds.

\section{c) Other Dynamical Processes}

A third proposal, by Boland and de Jong (1982, also 1984) is that the carbon abundance in a dense dark cloud is determined by the balance between the freezing of gas onto grains and the evaporation of grain mantles by the interstellar radiation field. Since there is no interstellar radiation in the center of dark clouds and the grain temperatures are very low, much of the gas should be frozen into grain mantles. In order to explain the incomplete depletion of heavy elements in the gas phase, they proposed that there must exist a dynamic process (turbulence) which carries cells of gas to the cloud surface on nearly the same time scale as that for freezeout. At the cloud surface the grain mantles would be evaporated by some as yet undetermined process, renewing the heavy element supply in the gas phase. At the same time, a considerable abundance of $\mathrm{C}_{\mathrm{I}}$ would be produced by UV dissociation of $\mathrm{CO}$ in gas cells which are then carried toward the cloud core by turbulence. Although in 1982 they predicted C I to CO ratios of order unity in cloud cores, in their 1984 model the $C_{I}$ was restricted to the outer $2 \mathrm{mag}$ of extinction. Thus the results of their latest version resemble in structure the early models without turbulent convection (e.g., Langer 1976a) although the total amount of $\mathrm{C}_{\mathrm{I}}$ they predict is much larger.

Williams and Hartquist (1984) criticized Boland and de Jong's theory and suggested as an alternative that the evaporation of grain mantles is due to sputtering in periodically recurring shock waves which are created by a process similar to that proposed by Norman and Silk (1980). The consequent recycling of mantle material into the gas phase enhances the $\mathrm{C}_{\mathrm{I}}$ abundance for about $10^{5} \mathrm{yr}$ after the shock passage. Unfortunately, in the best known shocked region, that in OMC-1, we have seen no evidence for emission from $\mathrm{C}_{\mathrm{I}}$ (Beichman et al. 1982). However, because of the high velocity of the shock in OMC-1, $\sim 37 \mathrm{~km} \mathrm{~s}^{-1}$ (Draine and Roberge 1982; Chernoff, Hollenbach, and McKee 1982), the temperature of the neutral gas peaks at $\sim 3000 \mathrm{~K}$. As pointed out by D. A. Williams $(1985$, private communication), this temperature is high enough to initiate reactions of $\mathrm{C}$ with $\mathrm{H}_{2}$, thus destroying the atomic carbon. A better place to look for carbon produced by this process would be in less violently shocked regions such as those near T Tauri stars.

\section{d) Internal Photon Production}

Photon production within clouds is implicit in many models. For example, Norman and Silk (1980) calculated that the UV flux which could be produced by shock waves initiated by mass-losing T Tauri stars within dark clouds is about $10^{-4}$ times the flux in the general interstellar medium. This, they claimed, is enough to provide a significant photodissociation rate in the interiors of dark clouds and prevent molecular abundances from being driven to their calculated equilibrium values. The UV field could also help to evaporate grain mantles, maintaining a large gas phase abundance of molecules in addition to $\mathrm{H}_{2}$. This process would presumably act in addition to the grain mantle sputtering process proposed by Williams and Hartquist (1984).

A similar idea, proposed by Prasad and Tarafdar (1983), is that inside dark clouds there may exist a dilute flux of UV photons which is produced by cosmic ray excitation of the Lyman and Werner systems of the hydrogen molecule. Subsequent deexcitation of the $\mathrm{H}_{2}$ molecule with the release of $\mathrm{UV}$ radiation acts to dissociate $\mathrm{CO}$, producing $\mathrm{C}$ I throughout the cloud. This process is capable of producing a very uniform abundance of $\mathrm{C}$ I even in the cores of dark clouds without embedded stars.

Montemerle et al. (1983) have shown that a majority of the T Tauri stars and other pre-main-sequence stars embedded in the $\rho$ Ophiuchi dark cloud have X-ray emission. Krolik and Kallman (1983) calculated the effect that this emission can have on the ionization and chemical equilibria within clouds. They showed that the inclusion of X-ray ionization into chemical equilibrium models can boost the neutral carbon abundance by two orders of magnitude although the absolute amount of carbon they obtained was still too low. However, if one combined this process with the related ones proposed by Williams and Hartquist (1984) and Norman and Silk (1980), one possibly could obtain a significant $\mathrm{C}$ I enhancement from $\mathrm{T}$ Tauri stars. If the embedded $\mathrm{T}$ Tauri stars do provide the mechanism for enhancing the $\mathrm{C}_{\mathrm{I}}$ abundance then one should not expect to see any $\mathrm{C}_{\mathrm{I}}$ in dark clouds without such stars.

\section{e) $\mathrm{C} / \mathrm{O}$ Greater than 1}

A fifth possibility concerns the gas phase $\mathrm{C}$ to $\mathrm{O}$ ratio in dense clouds. A value greater than 1 for this ratio was suggested by Tarafdar, Prasad, and Huntress (1983) who found from Copernicus observations that even in diffuse clouds the ratio of $\mathrm{C}$ to $\mathrm{O}$ varies from less than 1 to greater than 1. (This result must be regarded as tentative because of the high degree of saturation of the $\mathrm{C}$ II lines used in the Copernicus studies; A. E. Glassgold 1985, private communication). If the $\mathrm{C}$ to $\mathrm{O}$ ratio in dense clouds were greater than 1, Langer et al. (1984) have shown that then an excess of atomic carbon would be left after the formation of CO. In dense clouds one might expect considerable depletion of gas phase oxygen due, for example, to the absorption of $\mathrm{H}_{2} \mathrm{O}$ onto the grains. Some theoretical support for this comes from the work of Prasad and Huntress (1982) who inferred from their models of sulfur chemistry that the abundance of atomic oxygen is low in dense clouds. Moreover Herbst (1983) and Leung, Herbst, and Huebner (1984) required a large abundance of atomic carbon in the cores of 
clouds in order to reproduce in their models the observed abundance of complex hydrocarbons.

\section{f) Photodissociation}

Finally, in a photodissociation model similar to the earliest ones (e.g., Langer 1976a; Clavel, Viala, and Bel 1978), Tielens and Hollenbach $(1985 a b)$ have suggested that the entire observed column density of neutral carbon can be produced in a photodissociation region at the edges of molecular clouds. One difference between this model and earlier ones is that this model incorporates an enhanced (by a factor, $G_{0}$, of $10^{3}-10^{6}$ ) UV radiation field such as would be appropriate for the interface between a luminous $\mathrm{H}$ II region and a molecular cloud. Other major differences are a higher range of densities $\left(10^{3}-10^{6}\right.$ $\mathrm{cm}^{-3}$ ), a higher total carbon abundance and $\mathrm{C}$ to $\mathrm{O}$ ratio $(\mathrm{C} / \mathrm{O}=0.6$ compared to $\sim 0.4$ ) in the gas phase, and a more complete chemical code. This model is able to produce large $\mathrm{C}$ I column densities within $\sim 10 \mathrm{mag}$ of visual extinction from the $\mathrm{H}$ II region. However, when an estimation of the effects of CO self-shielding are included, the calculated column density of $\mathrm{C}$ I drops by a factor of about 2.

The observations presented here of the edge-on $\mathrm{H}$ II regions M 17 and S140 are meant to test a theory such as this last one, to determine whether the observed large C I abundance exists only at the edges of molecular clouds, particularly near ionization fronts, or if it is spread more generally throughout the clouds.

\section{THE OBJECTS}

\section{a) $M 17$}

The object M17 is thought to have a geometry similar to that of the Orion Nebula (M42) but turned $90^{\circ}$ from the line of sight (Gull and Balick 1974). That is, it is an ionization region eating its way into a large molecular cloud and forming a bowl-shaped interface. The bottom of the bowl is toward the southwest, and the sides are in the foreground and background. The luminosity of $\mathrm{M} 17$, as measured in the farinfrared, is $\sim(6-7) \times 10^{6} L_{\odot}$ (Harper et al. 1976; Wilson et al. 1979). It is ionized by a star cluster which is visually obscured but which is revealed in $0.9 \mu \mathrm{m}$ photographs (Beetz et al. 1976). The luminosity that can be accounted for by known stars is $\sim 3 \times 10^{6} L_{\odot}$ (Chini, Elsässer, and Neckel 1980). M17 is at a distance of $\sim 2.2 \mathrm{kpc}$ (Crampton, Georgelin, and Georgelin 1978; Chini, Elsässer, and Neckel 1980; see also Jaffe 1980). The molecular cloud southwest of M17 is an extremely long and massive one, extending over $4^{\circ}(\sim 150 \mathrm{pc}$; Elmegreen and Lada 1976; Elmegreen, Lada, and Dickinson 1979). The region studied in this paper is only a tiny fraction of the whole cloud in area but is by far the most luminous portion.

That the core of M17 is very dense is shown by observations of the CS molecule by Liszt and Linke (1975), Linke and Goldsmith (1980), Snell et al. (1984), and Mundy (1984). From $J=1 \rightarrow 0$ and $2 \rightarrow 1$ rotational transitions of $C S$, Linke and Goldsmith (1980) derived a cloud density of $n\left(\mathrm{H}_{2}\right)=6 \times 10^{4}$ $\mathrm{cm}^{-3}$, but from observations of higher transitions $(2 \rightarrow 1$ to $5 \rightarrow 4)$, Snell et al. (1984) and Mundy (1984) concluded that, over a $3^{\prime}$ diameter region centered on the cloud core, the emission from CS arises from clumps with density $n\left(\mathrm{H}_{2}\right) \approx 5 \times 10^{5}$ $\mathrm{cm}^{-3}$ with volume filling factor of $\sim 0.03$. Also, from observations of many transitions of $\mathrm{H}_{2} \mathrm{CO}$ at several positions in M17, S140, and NGC 2024, Mundy (1984) concluded that the density of foreground gas or interclump medium is probably $\gtrsim 6 \times 10^{4} \mathrm{~cm}^{-3}$, in agreement with the measurements of Linke and Goldsmith (1980) which were more sensitive to the lower density gas.

For the interpretation of the observations presented in this paper it is important to consider whether the normal to the ionization front lies in the plane of the sky. From models of the geometry of the far-infrared and radio continuum emission of M17, Icke, Gatley, and Israel (1980) have estimated that the normal to the southern ionization front (the one discussed in this paper) is $\sim 70^{\circ}$ from our line of sight; Felli, Churchwell, and Massi (1984) reached the same conclusion based on a recent high-resolution radio continuum map. This is so close to being in the plane of the sky that for the purposes of this paper the difference can be neglected. There is no evidence in the radio continuum map of M17 to suggest a hidden ionization front but if there were one, for example consisting of lower density ionized matter behind the main molecular cloud, the far-infrared continuum radiation should be a good indicator of where this heated material would lie. In both the farinfrared maps of M17 by Gatley et al. (1979) and Wilson et al. (1979) it can be seen that the far-infrared intensity falls very rapidly with distance into the molecular cloud. Moreover Gatley et al. showed that there is a strong temperature gradient in the far-infrared radiation along the same path, with the temperatures being highest in the ionization front, as defined by the radio continuum observations, and falling sharply away from it. This is consistent with the known ionizing stars dominating the heating and ionization of the $\mathrm{H}$ II region and molecular cloud, as shown by Icke, Gatley, and Israel (1980). It therefore appears that the influence of a hypothetical hidden ionization front must be small.

In order to compare our observations with theoretical models of photoionization regions, it is necessary to estimate the amount of ultraviolet flux at the ionization front. Because many of the exciting stars of M17 have very large extinctions and their characteristics are not well determined, it is not easy to directly estimate the stellar ultraviolet flux. Instead if we make the simple assumption that all the far-infrared flux of M17 was initially emitted by the ionizing stars in the ultraviolet we will find it possible to make this estimation. Wilson et al. (1979) measured a $69 \mu \mathrm{m}$ flux density $\left(F_{v}\right)$ of $1.2 \times 10^{-21} \mathrm{~W}$ $\mathrm{m}^{-2} \mathrm{~Hz}^{-1}$ from a 2.3 by 3.5 source elongated along the ionization front but lying between the molecular cloud peak and the ionization front. The integrated flux of this feature (given approximately by $v F_{v}$ ) is about $5.2 \times 10^{-9} \mathrm{~W} \mathrm{~m}^{-2}$. At a distance of $2.2 \mathrm{kpc}$ this source alone, excluding any background extended component, has a luminosity, $L$, of about $3.0 \times 10^{32}$ W or $7.8 \times 10^{5} L_{\odot}$. The embedded Kleinmann-Wright object (Kleinmann and Wright 1973) is thought to have a much lower luminosity than that, probably at most $2.5 \times 10^{4} L_{\odot}$ (Tokunaga and Thompson 1979), therefore we neglect it hereafter in our discussions. The length of the ionization front as defined by the far-infrared feature is 3.5 or $6.9 \times 10^{16} \mathrm{~m}$. If the molecular cloud core as viewed from the ionizing source has a circular cross section then its absorbing area, $A$, is $3.8 \times 10^{33}$ $\mathrm{m}^{2}$ and the flux passing through that area from the ionizing sources must be $L / A$ or $8.0 \times 10^{-2} \mathrm{~W} \mathrm{~m} \mathrm{~m}^{-2}$. In units of $1.6 \times 10^{-6} \mathrm{~W} \mathrm{~m}^{-2}$ (used by Tielens and Hollenbach) this is an ultraviolet enhancement factor, $G_{0}$, of $5 \times 10^{4}$.

\section{b) $S 140$}

$\mathrm{S} 140$ is an ionization front on one rim of the dark cloud L1204. It is excited by the star HD 211880 (B0 V, Crampton 
and Fisher 1974; $L \approx 5 \times 10^{4} L_{\odot}$, Panagia 1973), which lies $7^{\prime}$ to the SW. Embedded in the dark cloud is a cluster of infrared sources with a luminosity of $\sim 1.5 \times 10^{4} I_{\odot}$ (Harvey, Campbell, and Hoffman 1978; Beichman, Becklin, and WynnWilliams 1979; Schwartz et al. 1983) assuming S140 is at a distance of $0.91 \mathrm{kpc}$ (Crampton and Fisher 1974). Two members at least of this cluster have small ionization regions consistent with their being early B-type stars (Schwartz et al. 1983). Knapp et al. (1976) have observed carbon recombination line emission from S140 and have concluded that it arises in the visible ionization front rather than in a higher density region near the embedded sources. It appears that the embedded sources contribute most of the cloud luminosity but that HD 211880 dominates the ionization processes.

The core of S140 has also been studied in CS by Snell et al. (1984) and Mundy (1984) and in addition by Hayashi et al. (1985). The conclusions for the density structure of S140 are very similar to those for M17. The density, as measured with the high-level CS lines is $3-9 \times 10^{5} \mathrm{~cm}^{-3}$ over a $3^{\prime}$ diameter region with an estimated volume filling of 0.1 .

We can estimate the radiation field at S140 much more directly than we could for M17 since we know the spectral type and location of the ionizing star. From a model atmosphere for a B0 V star by Hickok and Morton (1968) as tabulated by Bradley and Morton (1969) and from stellar parameters tabulated by Panagia (1973) we calculate that a B0 V star emits about $10^{31} \mathrm{~W}$ at wavelengths between 912 and $2000 \AA$. At the projected distance of the ionization front of $7^{\prime}$ or $1.85 \mathrm{pc}$, this gives a flux of $2.4 \times 10^{-4} \mathrm{~W} \mathrm{~m}^{-2}$ or $G_{0}=150$.

\section{OBSERVATIONS}

\section{a) $\mathrm{C}_{\mathrm{I}}$}

The frequency of the ${ }^{3} P_{1} \rightarrow{ }^{3} P_{0}$ transition of $C_{I}$ was measured by Saykally and Evenson (1980) to be $492.1623 \mathrm{GHz}$ with an uncertainty of $0.7 \mathrm{MHz}$. However in our observations of many astronomical sources, we find the $\mathrm{C}$ I lines to be systematically displaced by $1.0 \mathrm{~km} \mathrm{~s}^{-1}$ relative to other spectral lines. This is particularly noticeable in dark clouds where the velocities of very narrow $\mathrm{C}$ I and $\mathrm{C}^{18} \mathrm{O}$ lines can be compared (Frerking et al. 1985). Therefore we believe that a more accurate value for the line frequency is $492.1607 \mathrm{GHz}$, and we use this frequency throughout this paper. This frequency was not determined from data contained in this paper.

The observations of this line of $\mathrm{C}_{\mathrm{I}}$ were made on four observing runs between 1981 August and 1984 July with the $0.9 \mathrm{~m}$ telescope of NASA's Kuiper Airborne Observatory at an altitude of $12.5 \mathrm{~km}$. We used an InSb hot-electron-bolometer heterodyne receiver similar to that described by Phillips and Jefferts (1974). The $1 \mathrm{MHz}$ bandwidth $\left(0.61 \mathrm{~km} \mathrm{~s}^{-1}\right)$ of the receiver was stepped along the spectrum by sweeping the klystron local oscillator under computer control. The receiver noise temperature varied from $\sim 500 \mathrm{~K}$ in 1981 to $\sim 350 \mathrm{~K}$ in 1983 and 1984. Each source was observed on two separate occasions, the results were consistent and were averaged. The beamsize for the observations is $\sim 3^{\prime}$; we estimate the pointing uncertainties to be $\$ 0.7$.

We calibrated with observations of $290 \mathrm{~K}$ and $80 \mathrm{~K}$ loads to determine the receiver temperature and with observations of the Moon to determine the beam efficiency. We assumed the temperature of the subsolar point to be $390 \mathrm{~K}$ and the submillimeter emissivity to be $97 \%$ (Linsky 1973). The measured beam efficiency varied from $32 \%$ in 1981 to $52 \%$ in 1983 and
1984. All values quoted for $T_{A}^{*}$ in this paper have been corrected for the beam efficiency in accordance with the original definition of $T_{A}{ }^{*}$ by Phillips, Jefferts, and Wannier (1973). They are thus equivalent to $T_{R}{ }^{*}$ as defined by Kutner and Ulich (1981). We usually cannot measure the aperture efficiency because of the difficulty in finding a point source bright enough to measure in a $1 \mathrm{MHz}$ bandwidth (e.g., Jupiter) at arbitrary times of year from a platform which is restricted in duration of observations and location of objects. We did, however, observe Jupiter in 1983 on a flight during which we also observed M17 for the second time. Assuming a temperature of $160 \mathrm{~K}$ for Jupiter at $609 \mu \mathrm{m}$ (Hildebrand et al. 1985), the aperture efficiency was $\sim 20 \%$. We estimate our absolute calibration uncertainty to be of the order of $25 \%$. Relative calibrations have less uncertainty.

\section{b) $\mathrm{CO}$}

We observed the $J=1 \rightarrow 0$ line of ${ }^{12} \mathrm{CO}$ and ${ }^{13} \mathrm{CO}$ in 1982 August and 1983 October with one of the $10.4 \mathrm{~m}$ telescopes at the Owens Valley Radio Observatory. The receiver noise temperature was $\sim 600 \mathrm{~K}(\mathrm{ssb})$ in 1982 and $\sim 230 \mathrm{~K}(\mathrm{ssb})$ in 1983. The backend was a $100 \mathrm{MHz}$ bandwidth acousto-optic spectrometer (Masson 1982) with $200 \mathrm{kHz}\left(\sim 0.5 \mathrm{~km} \mathrm{~s}^{-1}\right)$ resolution. At the frequencies of these transitions the telescope beam size is $\sim 1^{\prime}$. The pointing errors are $<0^{\prime} .5$ for these observations. We calibrated the $\mathrm{CO}$ spectra with an ambient temperature chopper and observations of the Moon. We assumed the temperature of the subsolar point to be $330 \mathrm{~K}$ at these frequencies. The measured beam efficiency was $\sim 85 \%$. Again we estimate absolute calibration uncertainties to be of order $25 \%$.

Thronson and Lada (1983) have shown that for sources with sharp intensity gradients, such as M17 and S140, the location of the observed peak intensity depends on the beam size used for the observations. For example, the peak $\mathrm{CO}$ intensity in M17 measured with a 1'.1 beam (Thronson and Lada) is $1^{\prime}$ NW of the peak measured with a 2'.3 beam (Lada 1976). Therefore in comparing two sets of observations of sources such as these, it is essential that they have comparable spatial resolution. To ensure this we have synthesized a $\mathrm{CO}$ beam that is approximately the same as the larger $\mathrm{C}$ I beam. At each position observed in the $\mathrm{C}$ I line we made a five point $\mathrm{CO}$ map with $1^{\prime}$ spacing and averaged the resulting spectra.

\section{c) $M 17$}

Figure 1 shows the $C_{1}$ positions observed in M17 superposed on (left) the $21 \mathrm{~cm}$ continuum map of Felli, Johnston, and Churchwell (1980) and (right) the CO map of Thronson and Lada (1983). The observed positions lie on a line roughly perpendicular to the ionization front (position angle of $53^{\circ}$ ) passing through the peak of the molecular line emission (Lada 1976, measured with a beam size comparable to ours, 2'3). We refer to the positions by their radial offset (in minutes of arc) from the reference position [0.0] whose coordinates are given in Table 1. This reference position is very close to the position of the Kleinmann-Wright object. Negative positions lie to the southwest, positive positions to the northeast. Position [0.0] lies on the large beam $\mathrm{CO}$ peak while position [2.5] lies in the ionization front as revealed by the $21 \mathrm{~cm}$ continuum. The five points near the molecular cloud peak were observed with roughly half beam spacings (1'25), the southwestern points were observed with nearly full beam spacings $(2 \cdot 5)$. 


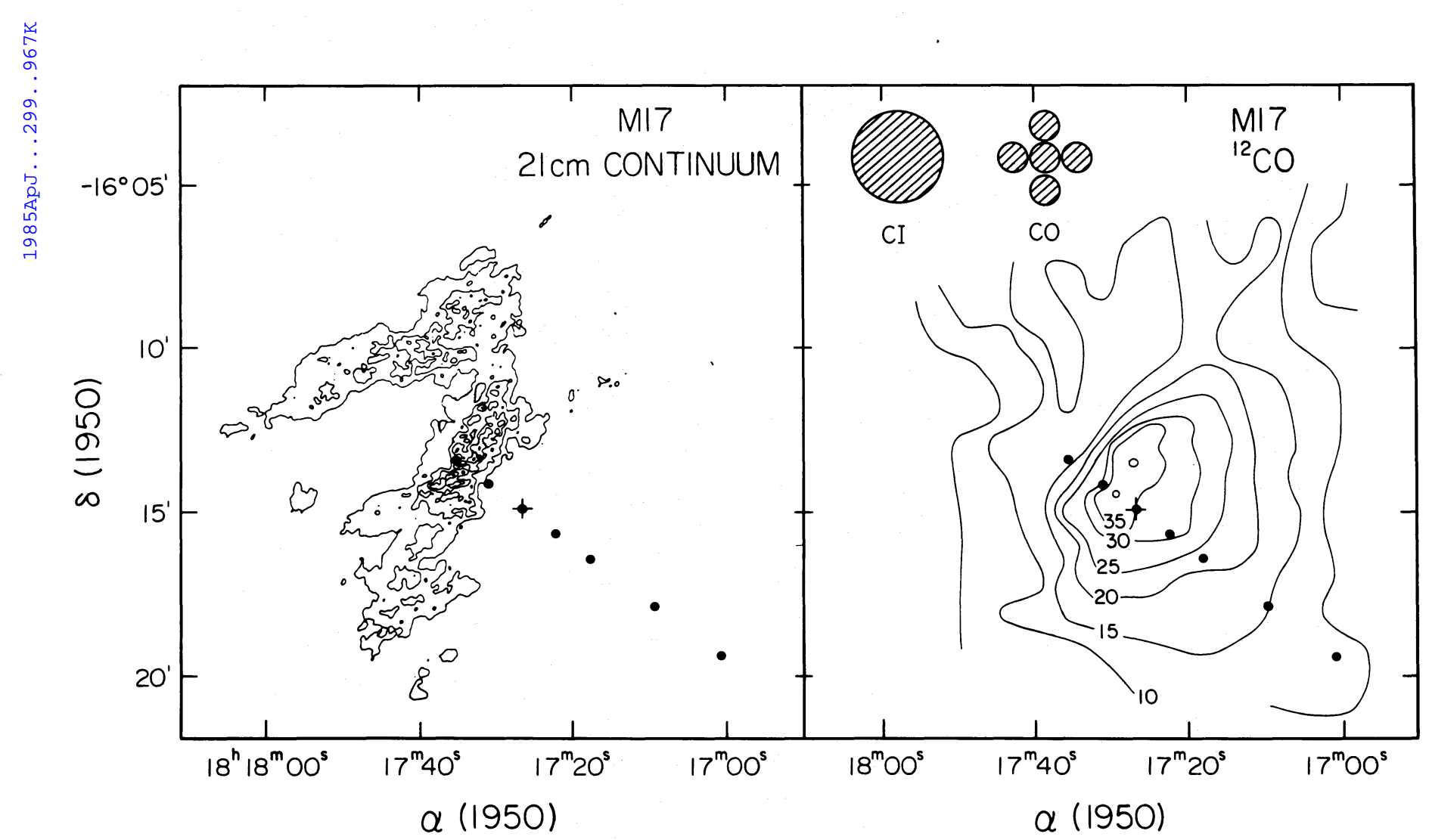

FiG. 1. - (left) The distribution of $21 \mathrm{~cm}$ continuum in M17 as shown in a VLA map (Felli, Johnston, and Churchwell 1980). (right) The distribution of peak $T_{*}^{*}(\mathrm{CO})$ measured with a 1'1 beam by Thronson and Lada (1983). Also shown are the positions of the $C_{1}$ measurements with the $C \mathrm{I}$ beam size and our synthesized CO beam. Position [0.0], marked with a cross, is the position of the peak CO emission when measured with a larger beam (2'3; Lada 1976).



Fig. 2. - left) The distribution of $21 \mathrm{~cm}$ continuum in S140 as shown in a Westerbork map (Falgarone and Gilmore 1981). The ionization front is heavily overresolved in this map. The ionization source is the star HD 211880, indicated on the map. Also shown are the C I beam size and our synthesized CO beam. (right) The distribution of peak $T_{A}^{*}(\mathrm{CO})$ observed with a 2'3 beam by Blair $e$ t al. (1978). The positions of the $\mathrm{C}$ I measurements are indicated by dots. Position [0.0], marked with a cross, is near the position of a bright infrared source (IRS 1). 
TABLE 1

M17 ObSERVEd Line Parameters



\section{d) $S 140$}

Figure 2 shows the positions observed in S140 superposed on (left) the $21 \mathrm{~cm}$ continuum map of Falgarone and Gilmore (1981) and (right) the ${ }^{12} \mathrm{CO}$ map of Blair et al. (1978). Again, the observed positions lie on a line nearly perpendicular to the ionization front (position angle of $45^{\circ}$ ) passing through the molecular cloud peak. Position [0.0] lies on the infrared source (Blair et al. 1978; Beichman, Becklin, and Wynn-Williams 1979) and position $[-1.4]$ lies within the ionization front as revealed by the radio continuum map. The observations were spaced by approximately full beamwidths $\left(2{ }^{\prime} 8\right)$ to the northeast of the peak but by only a half-beamwidth (1:4) to the southwest. The CO map of Blair et al. (1978) has an error in absolute position. The peak intensity should coincide with position [0.0] and the infrared source (Snell et al. 1984).

\section{RESULTS}

a) $M 17$

The observations of M17 are summarized in Table 1, and the spectra are shown in Figure 3. The $\mathrm{CO}$ profiles are the averages over five points spaced by $1^{\prime}$ as discussed above and illustrated in Figure 1. The ${ }^{12} \mathrm{CO}$ and ${ }^{13} \mathrm{CO}$ intensities peak sharply at $[0.0]$ where the antenna temperatures $\left(T_{A}{ }^{*}\right)$ are $39 \mathrm{~K}$ and $17 \mathrm{~K}$, respectively. The $\mathrm{C}$ I and ${ }^{13} \mathrm{CO}$ spectra are plotted on the same scale; the ${ }^{12} \mathrm{CO}$ spectra have been divided by a factor of 3. The $\mathrm{C}$ i spectral points are spaced by $1 \mathrm{MHz}$ but have been Hanning smoothed to an effective resolution of 2 $\mathrm{MHz}\left(\sim 1.2 \mathrm{~km} \mathrm{~s}^{-1}\right)$.

One striking aspect of these spectra is the similarity in shape and velocity between the $\mathrm{C} I$ lines and the ${ }^{13} \mathrm{CO}$ lines where the signal-to-noise ratio is high. Another striking aspect is that the $\mathrm{C}$ I line temperature peaks farther into the molecular cloud from the ionization front than does either of the $\mathrm{CO}$ lines. This is displayed graphically in Figure 4 which shows the line temperature integrated over velocity as a function of position. The integrated temperature of the $\mathrm{C}$ I line is higher than that of ${ }^{13} \mathrm{CO}$ from the position of the $\mathrm{C}$ I peak nearly as far into the dark cloud as we observe it, 7.5 from the $\mathrm{CO}$ peak.

Column densities of $\mathrm{C}$ I and $\mathrm{CO}$ were calculated using a standard LTE method as described in the Appendix. The estimates for M17 are shown in Table 2. These data show that the $\mathrm{C}$ I to $\mathrm{CO}$ ratio is small near the molecular peak but rises to a value $\gtrsim 0.1$ inside the dark cloud behind the front. The $\mathrm{C}_{\mathrm{I}}$ column density in M17 reaches its peak value about 1'3 $( \pm 0,7)$ farther from the ionization front than does the $\mathrm{CO}(0.8 \mathrm{pc}$ at a distance of $2.2 \mathrm{kpc}$ ). The CO peak itself is displaced from the ionization front by a similar amount. The peak $\mathrm{C}_{\mathrm{I}}$ column density measured with our beam is therefore $\sim 1.6 \mathrm{pc}(\sim 2,5)$ from the ionization front. If the $\mathrm{C}$ I and $\mathrm{CO}$ peaks lie along a line normal to the front (i.e., in a plane perpendicular to our line of sight), the amount of extinction between the $\mathrm{C}_{\text {I }}$ peak and the front can be estimated. The map of ${ }^{13} \mathrm{CO}$ column density by Thronson and Lada (1983) shows that the column density of the $\mathrm{CO}$ peak is $\gtrsim 3 \times 10^{17} \mathrm{~cm}^{-2}$ over a nearly circular region $\sim 3^{\prime}$ in diameter and peaking within $1^{\prime}$ of our position $[0]$. This is in satisfactory agreement with our measurements in a $\sim 3^{\prime}$ synthesized beam from which we derive that $N\left({ }^{13} \mathrm{CO}\right)=2.5 \times 10^{17} \mathrm{~cm}^{-2}$ If the $\mathrm{CO}$ core is roughly spherical with an extent along the line of sight similar to that in the plane of the sky (see Fig. 5), the visual extinction between the ionization front and the $\mathrm{C}$ I peak is $\sim 100$, assuming $A_{v}=$ $4 \times 10^{-16} N\left({ }^{13} \mathrm{CO}\right)$. This corresponds to an average $\mathrm{H}_{2}$ density of $\sim 3 \times 10^{4} \mathrm{~cm}^{-3}$ between the front and the $\mathrm{C}$ I peak. From

TABLE 2

M17 Derived Column Densities

\begin{tabular}{|c|c|c|c|c|c|c|c|}
\hline $\begin{array}{c}\text { Offset } \\
\text { (arcmin) }\end{array}$ & $\begin{array}{c}T_{\text {ex }}(\mathrm{CO}) \\
(\mathrm{K})\end{array}$ & $\tau\left({ }^{13} \mathrm{CO}\right)$ & $\begin{array}{c}N\left({ }^{13} \mathrm{CO}\right) \\
\left(10^{16} \mathrm{~cm}^{-2}\right)\end{array}$ & $\tau(\mathrm{C} \mathrm{I})$ & $\begin{array}{c}N(\mathrm{C} \mathrm{I}) \\
\left(10^{16} \mathrm{~cm}^{-2}\right)\end{array}$ & $\frac{N(\mathrm{C} \mathrm{l})^{\mathrm{a}}}{N(\mathrm{CO})}$ & $A_{V}{ }^{\mathrm{b}}$ \\
\hline $2.5 .$. & $43^{\mathrm{c}}$ & 0.09 & 5.1 & 0.12 & 35. & 0.08 & \\
\hline $1.3 \ldots$ & $43 .^{c}$ & 0.34 & 15.6 & 0.17 & 37. & 0.03 & \\
\hline $0.0 \ldots \ldots \ldots \ldots$ & 43. & 0.56 & 24.9 & 0.34 & 77. & 0.03 & 100 \\
\hline$-1.3 \ldots \ldots \ldots \ldots$ & 37. & 0.42 & 14.7 & 0.54 & 109. & 0.08 & 59 \\
\hline$-2.5 \ldots \ldots \ldots \ldots$ & 31. & 0.28 & 6.8 & 0.57 & 73. & 0.12 & 27 \\
\hline$-5.0 \ldots \ldots \ldots \ldots$ & 21. & 0.33 & 3.0 & 0.58 & 50. & 0.19 & 12 \\
\hline$-7.5 .$. & 18. & 0.44 & 2.7 & 0.32 & 22. & 0.09 & 011 \\
\hline
\end{tabular}

${ }^{\text {a }}$ Assumes $N(\mathrm{CO})=90 N\left({ }^{13} \mathrm{CO}\right)$.

b Assumes $A_{V}=4 \times 10^{-16} N\left({ }^{13} \mathrm{CO}\right.$ Dickman 1978).

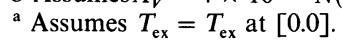






FIG. 3-Comparison of $\mathrm{C}_{\mathrm{I}}$ and $\mathrm{CO}$ line profiles in $\mathrm{M} 17$. The $\mathrm{C}_{\mathrm{I}}$ and ${ }^{13} \mathrm{CO}$ spectra are on the same scale; the ${ }^{12} \mathrm{CO}$ spectra have been divided by 3 . In this figure the ionization front lies to the left, approximately at position $[2.5]$. 


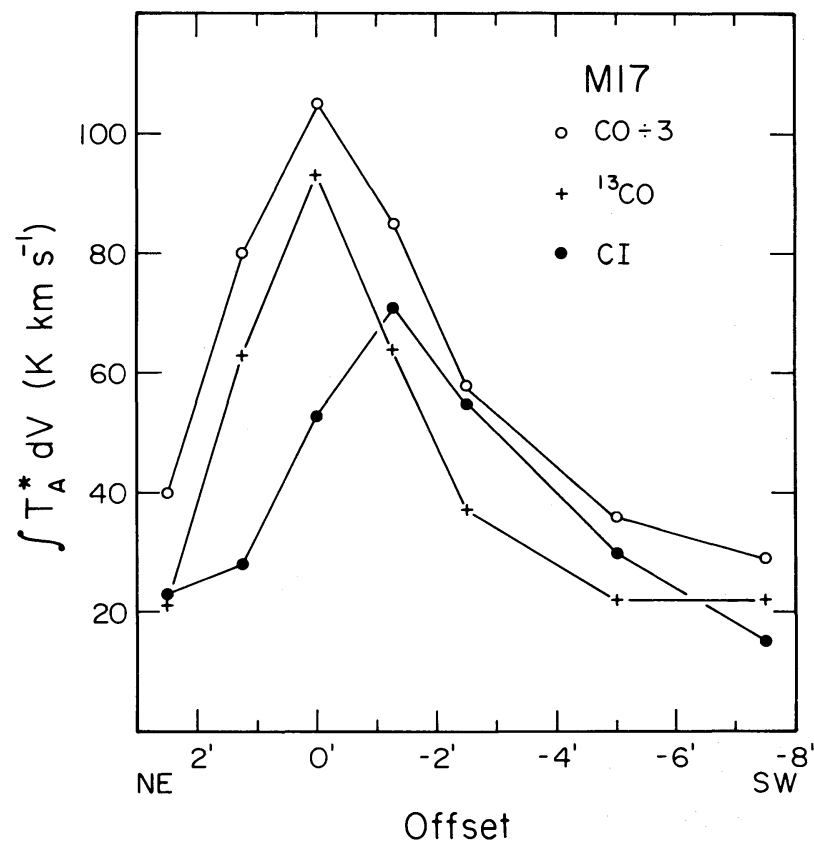

FIG. 4.- Comparison of $\mathrm{C} I$ and $\mathrm{CO}$ antenna temperatures in M17 integrated over velocity. The ionization front is again to the left. The $\mathrm{C}$ I and ${ }^{13} \mathrm{CO}$ are on the same scale; the ${ }^{12} \mathrm{CO}$ temperatures have been divided by 3 .

the observations of CS in M17 described in $\S$ III $a$ we conclude that the average density derived here is not excessively large.

The calculated $\mathrm{C}$ I to $\mathrm{CO}$ ratio at the position inside the ionization front (position [2.5]) is higher than the ratio near the molecular peak. However, there is a large uncertainty associated with the calculations for this position. The observed antenna temperature of $\mathrm{CO}$ inside the ionization front is low, $18 \mathrm{~K}$, yet it seems unlikely that the physical temperature is low. It seems more likely that the observed temperature is due to clumps of molecular gas with a low filling factor embedded in the ionization front. Such clumps would be similar to those seen by Felli, Churchwell, and Massi (1984) in the radio continuum. To explain the low ${ }^{12} \mathrm{CO}$ to ${ }^{13} \mathrm{CO}$ antenna temperature ratio, the clumps would have to be optically thick in ${ }^{12} \mathrm{CO}$. We have assumed that the excitation temperature at this position is the same as at the molecular peak. If there were an error in this assumed temperature it would affect the derivation of the $\mathrm{CO}$ column density more than that of the $\mathrm{C}$ I column density, if the temperature were higher than assumed here, the true $\mathrm{C}_{\mathrm{I}}$ to $\mathrm{CO}$ ratio would be lower than derived here.

b) $\$ 140$

The S140 observations are summarized in Table 3 , and the spectra are shown in Figure 6 . The $\mathrm{C}_{\mathrm{I}}$ and ${ }^{13} \mathrm{CO}$ spectra are plotted on the same scale, the ${ }^{12} \mathrm{CO}$ spectra have been divided by a factor of 3 . The $\mathrm{C}_{\mathrm{I}}$ spectral points are spaced by $1.5 \mathrm{MHz}$ $\left(\sim 0.9 \mathrm{~km} \mathrm{~s}^{-1}\right)$ and have not been smoothed.

Once again, although the lines in S140 are weaker than those in M17 and the signal-to-noise is correspondingly smaller, the $\mathrm{C}$ I line profiles show a strong resemblance to the ${ }^{13} \mathrm{CO}$ lines. The most notable feature of these spectra, however, is the weakness of the $\mathrm{C}$ I line at the $\mathrm{CO}$ peak. In fact, as shown by the integrated temperatures in Figure 7, the $\mathrm{C}$ I line does not have a peak position. Rather it gradually increases in intensity from the ionization front into the dark cloud. In the dark cloud the integrated temperature of the $\mathrm{C}$ I lines reaches a plateau and is the same as that of ${ }^{13} \mathrm{CO}$ throughout.

The calculated $\mathrm{C}_{\text {I }}$ column density (Table 4) also does not have a peak. It reaches a broad plateau $\sim 3^{\prime}$ into the cloud

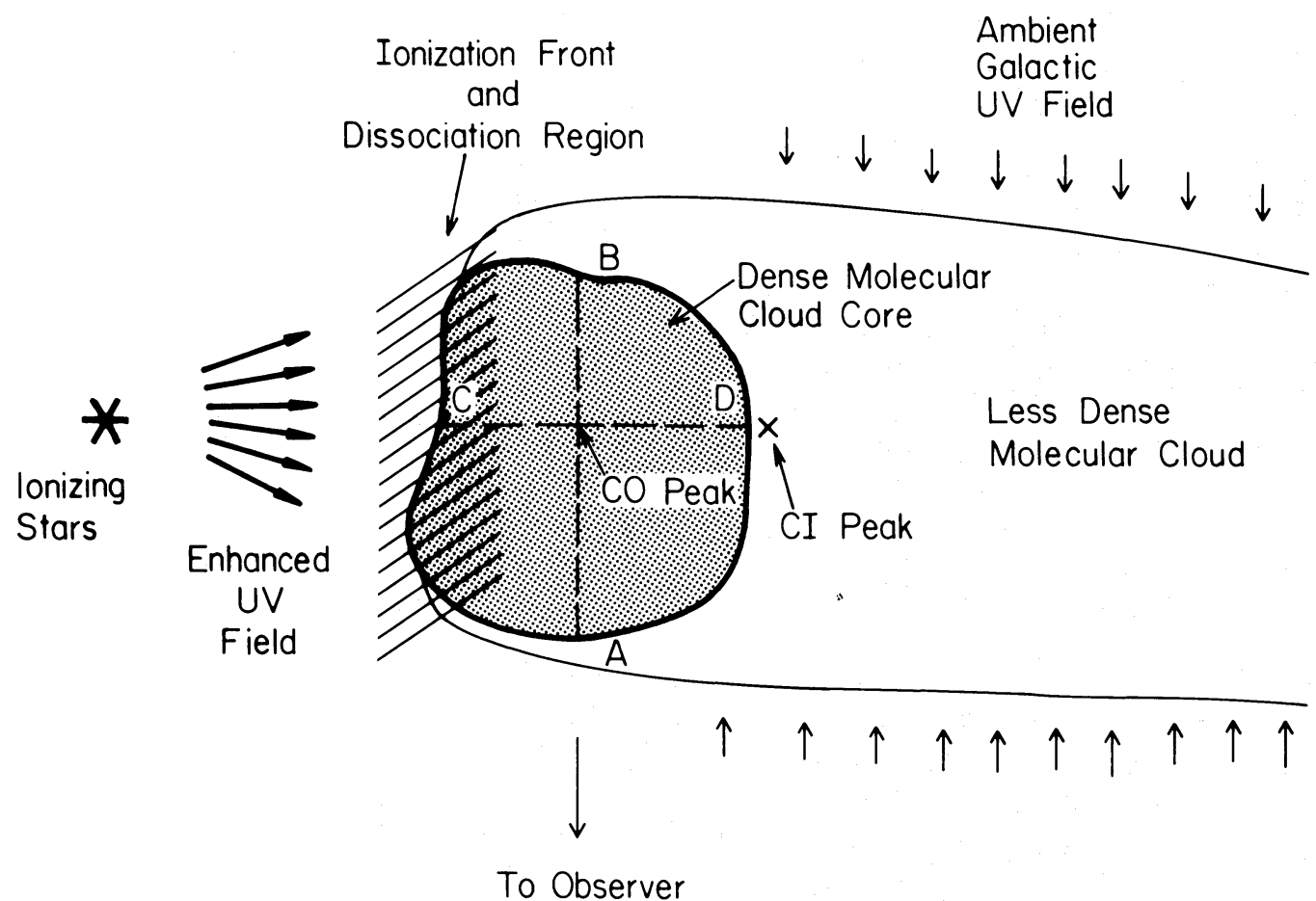

FIG. 5.-Schematic drawing of the geometry assumed for the calculation of extinction through the molecular cloud. We assume that the dense molecular condensation at the molecular cloud peak is roughly spherical and that the extinction as measured by the ${ }^{13} \mathrm{CO}$ column density through the peak (line $\mathrm{AB}$ ) is the same as that from the ionization front through the molecular peak to the carbon peak (line $\mathrm{CD}$ ). 
TABLE 3

S140 Observed Line Parameters

\begin{tabular}{|c|c|c|c|}
\hline $\begin{array}{c}\text { Offset } \\
\left({\text { arcmin })^{\mathrm{a}}}^{2}\right.\end{array}$ & $\begin{array}{l}T_{A}^{*} \\
(\mathrm{~K})\end{array}$ & $\frac{V_{\text {avg }}}{\left(\mathrm{km} \mathrm{s}^{-1}\right)}$ & $\begin{array}{c}\Delta V(\mathrm{FWHM}) \\
\left(\mathrm{km} \mathrm{s}^{-1}\right)\end{array}$ \\
\hline \multicolumn{4}{|c|}{$\mathrm{C}_{\mathrm{I}}$} \\
\hline  & $\begin{array}{l}1.8 \\
4.1 \\
6.5 \\
6.0 \\
4.8 \\
4.3\end{array}$ & $\begin{array}{l}-7.4 \\
-7.2 \\
-7.6 \\
-7.6 \\
-7.5 \\
-7.2\end{array}$ & $\begin{array}{l}3.7 \\
2.6 \\
3.7 \\
3.2 \\
5.1 \\
4.0\end{array}$ \\
\hline \multicolumn{4}{|c|}{${ }^{12} \mathrm{CO}$} \\
\hline 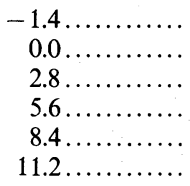 & $\begin{array}{r}5.1 \\
24.6 \\
15.9 \\
13.7 \\
12.7 \\
13.0\end{array}$ & $\begin{array}{l}-8.2 \\
-7.8 \\
-7.6 \\
-7.7 \\
-7.7 \\
-7.4\end{array}$ & $\begin{array}{l}3.1 \\
5.9 \\
4.8 \\
4.8 \\
5.0 \\
5.0\end{array}$ \\
\hline \multicolumn{4}{|c|}{${ }^{13} \mathrm{CO}$} \\
\hline $\begin{array}{r}-1.4 \ldots \ldots \ldots \ldots \\
0.0 \ldots \ldots \ldots \ldots \\
2.8 \ldots \ldots \ldots \ldots \\
5.6 \ldots \ldots \ldots \ldots \\
8.4 \ldots \ldots \ldots \ldots \\
11.2 \ldots \ldots \ldots \ldots\end{array}$ & $\begin{array}{r}1.6 \\
12.6 \\
8.3 \\
7.0 \\
5.8 \\
6.4\end{array}$ & $\begin{array}{l}-7.4 \\
-7.5 \\
-7.6 \\
-7.3 \\
-7.4 \\
-7.0\end{array}$ & $\begin{array}{l}2.9 \\
3.2 \\
3.1 \\
3.2 \\
3.2 \\
2.8\end{array}$ \\
\hline
\end{tabular}

${ }^{\mathrm{a}}$ Offset from $\alpha(1950)=22^{\mathrm{h}} 17^{\mathrm{m}} 42^{\mathrm{s}}, \delta(1950)=63^{\circ} 03^{\prime} 45^{\prime \prime}$ along a line at position angle $45^{\circ}$.

from the CO peak $(0.8 \mathrm{pc}$ at a distance of $910 \mathrm{pc})$. In the case of $\mathrm{S} 140$ our measurements of the column density of ${ }^{13} \mathrm{CO}$ are higher than those of Blair et al. (1978); we measure $N\left({ }^{13} \mathrm{CO}\right)=8 \times 10^{16} \mathrm{~cm}^{-2}$ in the synthesized beam near the peak while Blair et al. give a peak of $\sim 5 \times 10^{16} \mathrm{~cm}^{-2}$ in a smaller (1'.1) beam. If we use our value for $N\left({ }^{13} \mathrm{CO}\right)$ we find an extinction of $\sim 30 \mathrm{mag}$ between the ionization front and position [2.8] where the $\mathrm{C}$ I column density first attains its maximum value. This corresponds to an average $\mathrm{H}_{2}$ density of $\sim 10^{4} \mathrm{~cm}^{-3}$.

The discussion of the uncertainty involved in calculating the column densities in the ionized region in M17 holds also for S140.

\section{DISCUSSION}

\section{a) Comparison of Results with Photodissociation Model}

Our data show that the emission from $\mathrm{C}_{\mathrm{I}}$ is spread widely throughout molecular clouds and is strong even far from ionization fronts. We can exclude the possibility that $\mathrm{C}_{\mathrm{I}}$ exists mainly in dissociation regions on cloud edges fronting $\mathrm{H}$ II regions as suggested by Tielens and Hollenbach $(1985 a, b)$.

M17 in particular has one of the brightest $C_{1}$ lines yet observed. Its peak antenna temperature $(11 \mathrm{~K})$ is the same as that of Orion, and its line width $\left(6.4 \mathrm{~km} \mathrm{~s}^{-1}\right)$ is even greater than that of Orion $\left(5 \mathrm{~km} \mathrm{~s}^{-1}\right)$. In the Tielens and Hollenbach (1985a) model for Orion most of the C I line intensity is produced in a region within 10 mag of visual extinction of the ionization front, yet the position we observe from the peak $\mathrm{C} \mathrm{I}$ intensity in M17 is $\sim 3^{\prime}(1.8 \mathrm{pc})$ from the front. Even making allowances for pointing uncertainties and possible smearing due to our large beam, there are still probably more than 60 mag of visual extinction between the front and the $\mathrm{C}$ I peak. Also, the integrated $C_{1}$ intensity falls from the peak value by only a factor of 2 in the next $3.8(2.3 \mathrm{pc})$. We do not feel therefore that the special geometry of an ionization front which was invoked to explain previous Orion observations can account for our observations of M17 and likewise for those of S140.

One interesting feature of the Tielens and Hollenbach model is that the predicted column density of $\mathrm{C} I$ is independent of the magnitude of the UV radiation field for UV enhancement factors $\left(G_{0}\right)$ of $10^{3}-10^{6}$, and is about $4.5 \times 10^{17} \mathrm{~cm}^{-2}$ in their standard model. It is relevant to ask whether this same column density could be produced by the average interstellar field. Tielens and Hollenbach have not published such a calculation, but for a model cloud illuminated with the ambient UV field $\left(G_{0}=1\right)$ Boland and de Jong (1984) have calculated the same value for the column density of $\mathrm{C}$ I. (It must be noted, however, that production of this large column density in only 2 mag of extinction requires that one-third of all the carbon must be in the form of $\mathrm{C}_{\mathrm{I}}$ )

Although the derived column density may be independent of $G_{0}$, the depth at which the $\mathrm{C}_{\mathrm{I}}$ is produced must depend strongly on the penetration depth of the UV radiation. For example, in their model with $G_{0}=10^{5}$, Tielens and Hollenbach found that the $\mathrm{C}$ I was produced in the outer $10 \mathrm{mag}$ of extinction while models for photodissociation regions with $G_{0}=1$ (e.g., Boland and de Jong 1984) have shown that the neutral carbon region extends about 2 mag into the cloud. From this we can roughly estimate the depth of neutral carbon in other regions. In M17, where we estimate $G_{0}=5 \times 10^{4}$, the maximum depth of $C$ I should still be about 10 mag. In $\mathrm{S} 140$ where $G_{0} \approx 150$ the C I depth should only be about $6 \mathrm{mag}$. The extinctions we derive for the line of sight through the molecular cloud peak (100 for M17, 30 for S140) are so much larger than the calculated maximum $\mathrm{C}$ I depth that we conclude that the UV from the $\mathrm{H}$ II region cannot be the major source of $\mathrm{C}$.

TABLE 4

S140 Derived Column Densities

\begin{tabular}{cccccccc}
\hline \hline $\begin{array}{c}\text { Offset } \\
(\operatorname{arcmin})\end{array}$ & $\begin{array}{c}T_{\text {ex }}(\mathrm{CO}) \\
(\mathrm{K})\end{array}$ & $\tau\left({ }^{13} \mathrm{CO}\right)$ & $\begin{array}{c}N\left({ }^{13} \mathrm{CO}\right) \\
\left(10^{16} \mathrm{~cm}^{-2}\right)\end{array}$ & $\tau(\mathrm{C} \mathrm{I})$ & $\begin{array}{c}N(\mathrm{C} \mathrm{I}) \\
\left(10^{16} \mathrm{~cm}^{-2}\right)\end{array}$ & $\frac{N(\mathrm{C} \mathrm{I})^{\mathrm{a}}}{N(\mathrm{CO})}$ & $A_{V}{ }^{\mathrm{b}}$ \\
\hline$-1.4 \ldots \ldots \ldots \ldots$ & $28 .^{\mathrm{c}}$ & 0.07 & 0.8 & 0.11 & 9.0 & 0.13 & $\ldots$ \\
$0.0 \ldots \ldots \ldots \ldots$. & 28. & 0.71 & 8.0 & 0.26 & 15.1 & 0.02 & 32 \\
$2.8 \ldots \ldots \ldots \ldots$ & 19. & 0.73 & 3.9 & 1.06 & 45.8 & 0.13 & 16 \\
$5.6 \ldots \ldots \ldots \ldots$ & 17. & 0.71 & 3.1 & 1.41 & 42.6 & 0.15 & 12 \\
$8.4 \ldots \ldots \ldots \ldots$ & 16. & 0.61 & 2.4 & 1.13 & 52.4 & 0.24 & 10 \\
$11.2 \ldots \ldots \ldots \ldots$ & 16. & 0.68 & 2.4 & 0.88 & 34.1 & 0.16 & 10 \\
\hline
\end{tabular}

${ }^{a}$ Assumes $N(\mathrm{CO})=90 N\left({ }^{13} \mathrm{CO}\right)$.

${ }^{\mathrm{b}}$ Assumes $A_{V}=4 \times 10^{-16} N\left({ }^{13} \mathrm{CO}\right)$ (Dickman 1978).

c Assumes $T_{\mathrm{ex}}=T_{\mathrm{ex}}$ at $[0.0]$. 




Fig. 6.-Comparison of $\mathrm{C}$ I and $\mathrm{CO}$ line profiles in S140. The $\mathrm{C}$ I and ${ }^{13} \mathrm{CO}$ spectra are on the same scale; the ${ }^{12} \mathrm{CO}$ spectra have been divided by 3. In this figure the ionization front lies to the left, approximately at position [-1.4]. As in Fig. 3 , the ${ }^{12} \mathrm{CO}$ spectra are uppermost, the ${ }^{13} \mathrm{CO}$ spectra are in the middle, and the $\mathrm{C} I$ spectra are on the bottom. 


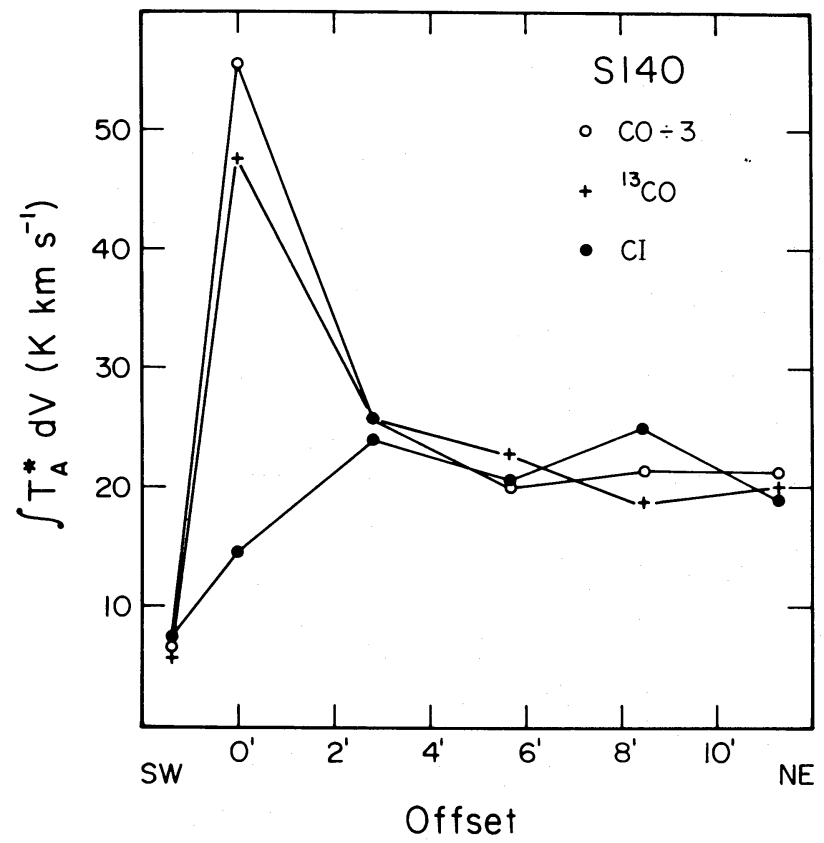

Fig. 7.-Comparison of $\mathrm{C} \mathrm{I}$ and $\mathrm{CO}$ antenna temperatures in $\mathrm{S} 140$ integrated over velocity. The ionization front is again to the left. The $\mathrm{C}_{\mathrm{I}}$ and ${ }^{13} \mathrm{CO}$ are on the same scale, the ${ }^{12} \mathrm{CO}$ temperatures have been divided by 3 .

There remains the more diffuse galactic UV radiation field to consider. Could it produce the $\mathrm{C}_{\mathrm{I}}$ observed in the dark cloud far from the $\mathrm{H}$ II region? To test this hypothesis we estimated the line-of-sight extinctions through the clouds from the ${ }^{13} \mathrm{CO}$ column density. These extinctions are listed in Tables 2 and 4 for M17 and S140, respectively. For positions farther from the ionization front than the $\mathrm{CO}$ peak, one can see that the ratio of $\mathrm{C}$ I to $\mathrm{CO}$ is about $0.1-0.2$ for values of $A_{v}$ from 10 to $60 \mathrm{mag}$. If the $\mathrm{C}$ I were concentrated in a shell only a few magnitudes thick on the surface of the cloud, one would expect that the absolute abundance of $\mathrm{C}$ I would be roughly constant and that the ratio of $\mathrm{C}$ I to $\mathrm{CO}$ would be inversely proportional to the CO column density or $A_{v}$. The data for M17, where the range in $A_{v}$ is large, are not consistent with there being a thin layer of $\mathrm{C}$ I around the cloud, but they are consistent, within the measurement errors, with a uniform ratio of $\mathrm{C}_{\mathrm{I}}$ to $\mathrm{CO}$.

The similarity in the shapes and widths of the $\mathrm{C}$ I and ${ }^{13} \mathrm{CO}$ lines is an indication that these lines arise from the same spatial regions and that $\mathrm{C}_{\mathrm{I}}$ is well mixed with the cloud. One exception to this rule is that $\mathrm{C}_{\mathrm{I}}$ appears to avoid the most dense and hot regions in molecular clouds, such as the $\mathrm{CO}$ peaks in M17 and $\mathrm{S} 140$. We believe that although some $\mathrm{C}$ I must be present in photodissociation regions on the edges of clouds as originally suggested by Langer (1976a) there must also be additional sources of $\mathrm{C}_{\mathrm{I}}$ within the clouds. Alternative proposals for production of $\mathrm{C}_{\mathrm{I}}$ summarized here in the Introduction, particularly those that produce it throughout the bulk of the cloud, must be considered.

\section{b) Other Models}

A particularly interesting theory is that of Langer et al. (1984) on the $\mathrm{C}$ to $\mathrm{O}$ ratio in dense clouds since it leads to a general chemical model which does not depend strongly on any specific parameter values of the cloud in question and which makes additional testable chemical predictions some of which seem to be borne out by observation. e.g., the lack of widespread $\mathrm{O}_{2}$ and thermal $\mathrm{H}_{2} \mathrm{O}$ in the gas phase. The calculations of Tarafdar et al. (1985) on the dynamical collapse of clouds is also promising since it fits our new data and previously existing data very well, but it does have the problem of requiring relatively young clouds. Several of the other proposals also lead to testable predictions. The feasibility of the suggestion of Prasad and Tarafdar (1983), that the internal UV field is capable of creating the observed $\mathrm{C} \mathrm{I}$, could be determined by calculating the near-infrared $\mathrm{H}_{2}$ line flux that would be produced by the same process. Putting limits on the observed infrared flux would than limit the efficiency of this process. Similarly, theories which predict production of $\mathrm{C}_{\mathrm{I}}$ near embedded T Tauri stars (Norman and Silk 1980; Krolik and Kallman 1983; Williams and Hartquist 1984) could be tested by observing dark clouds with and without $\mathrm{T}$ Tauri stars to see if $\mathrm{C}_{\mathrm{I}}$ is preferentially present near embedded stars. The remaining two proposals have problems. Time-dependentchemistry models need to be recalculated using new information on the dissociation of $\mathrm{H}_{3}{ }^{+}$. The theory of Boland and de Jong $(1982,1984)$ has the disadvantage that in its latest version it predicts that most of the $\mathrm{C}_{\mathrm{I}}$ will be in the outer $2 \mathrm{mag}$ of extinction. This is not supported by our data or those of Frerking et al. (1985) on the $\rho$ Ophiuchi dark cloud.

\section{c) Comparison with NGC 1977}

Wootten et al. (1982) have shown that in the case of NGC 1977 , a nearby $(\sim 500 \mathrm{pc})$ edge-on $\mathrm{H}$ II region, the $\mathrm{C}_{\mathrm{I}}$ has a sharp emission peak near the ionization front. The differences between these observations and those of Wootten et al. are in the spatial resolution and the size of the areas mapped. The map of NGC 1977 was made with a 45" beam, giving a spatial resolution of $0.1 \mathrm{pc}$. The $\mathrm{C}_{\mathrm{I}}$ peak near the ionization front showed up in only one map point. Our maps of M17 and S140 were made with $\sim 3^{\prime}$ resolution, giving spatial resolutions of $\sim 1.8 \mathrm{pc}$ in M17 and $0.8 \mathrm{pc}$ in S140. If M17 and S140 had sharp but small C I peaks similar to that in NGC 1977, we probably would not have detected them. However, our maps of M17 and S140 cover a much larger area than the NGC 1977 map. They show that $\mathrm{C}_{\mathrm{I}}$ is important throughout molecular clouds and that the bulk of it does not lie near the ionization front.

\section{d) Optical Depth Effects}

The maximum optical depth that we derive here for $\mathrm{C}_{\mathrm{I}}$ is 1.41 , sufficiently large to introduce errors caused by inhomogeneities in the cloud material. For such large optical depths Phillips and Huggins (1981; see also Phillips et al. 1979) devised a method of estimating the $\mathrm{C}_{\mathrm{I}}$ optical depths by comparing the $\mathrm{C}_{\mathrm{I}}$ line widths with those of several transitions and isotopic species of $\mathrm{CO}$. We do not have observations of enough isotopic species and transitions available to make a detailed comparison for M17 and S140 but based on the observed line widths of $\mathrm{C}_{\mathrm{I}}$ and ${ }^{13} \mathrm{CO}$ we estimate that the results for the ratio of $N(\mathrm{C}$ I $)$ to $N(\mathrm{CO})$ would be similar to those we have obtained using the line temperatures (Tables 2 and 4). The optical depths of the ${ }^{13} \mathrm{CO}$ lines can be checked by comparison with rarer isotopes. N. J. Evans (1984, private communication) has observed the $J=1 \rightarrow 0$ transition of $\mathrm{C}^{18} \mathrm{O}$ at the same positions we have observed in $\mathrm{S} 140$ and at the [0.0] position in M17. From the ratio of the $\mathrm{C}^{18} \mathrm{O}$ and ${ }^{13} \mathrm{CO}$ antenna temperatures we deduce that the ${ }^{13} \mathrm{CO}$ lines are optically thin $(\tau<1)$ 
e) The Relationship between $A_{V}$ and $\mathrm{CO}$ Column Density

The relationship between $A_{V}$ and $N\left({ }^{13} \mathrm{CO}\right)$ derived by Dickman [1978; $\left.A_{V}=4 \times 10^{-16} N\left({ }^{13} \mathrm{CO}\right)\right]$ and extrapolated here to derive large values of $A_{V}$ is almost certainly an oversimplification. For example Frerking, Langer, and Wilson (1982) have measured the relationships between visual extinction and the column densities of various isotopes of $\mathrm{CO}$ in two dark clouds, Taurus and $\rho$ Oph. In general they find highly nonlinear relations. However for the $\rho$ Oph cloud the $N\left({ }^{13} \mathrm{CO}\right)$ versus $A_{V}$ relationship of Dickman gives an excellent fit to the data for $1.7 \times 10^{16} \lesssim N\left({ }^{13} \mathrm{CO}\right) \lesssim 2.7 \times 10^{16} \mathrm{~cm}^{-2}\left(7 \lesssim A_{V} \lesssim\right.$ 11). They have no data for larger column densities; below the lower limit, Dickman's relationship provided only a lower limit to the visual extinction. In contrast, in the Taurus dark cloud the relationship gave only a lower limit to the extinction for all values of column density.

The dark clouds associated with M17 and S140 probably resemble the $\rho$ Oph cloud more closely than the Taurus cloud since they have excitation temperatures $\gtrsim 15 \mathrm{~K}$. Therefore, based on the results of Frerking, Langer, and Wilson (1982) we believe that the values we have estimated for $A_{V}$ in Tables 2 and 4 are quite possibly correct or are at least lower limits to the true extinction.

Bally and Langer (1982), Chu and Watson (1983), and Glassgold, Huggins, and Langer (1985) have discussed the effects of the self-shielding of $\mathrm{CO}$ from photodissociation on the relationships between the abundance of $\mathrm{CO}$ isotopes and visual extinction near the edges of molecular clouds. Many of the results of Frerking, Langer, and Wilson (1982) can be explained by isotope-selective photodestruction. However, this process is most important at low visual extinctions $\left(A_{V} \lesssim 2\right)$ and 4. If this is true in M17 and S140, then the values of $N(\mathrm{C} \mathrm{I}) / N(\mathrm{CO})$ we derived are also lower limits.

The ratio between $N\left({ }^{13} \mathrm{CO}\right)$ and $N(\mathrm{CO})$ can be affected also by isotopic fractionation. Near the edges of clouds the ratio of $N(\mathrm{CO})$ to $N\left({ }^{13} \mathrm{CO}\right)$ is probably lower than the value of 90 assumed in calculating the ratio of $N(\mathrm{C}$ I) to $N(\mathrm{CO})$ in Tables 2 and 4. If this is true in M17 and S140, then the values of $N(\mathrm{C} \mathrm{I}) / N(\mathrm{CO})$ we derived are also lower limits.

\section{f) Comparison of $\mathrm{C}$ I with $\mathrm{C}_{2} \mathrm{H}$}

$\mathrm{C}_{2} \mathrm{H}$ is another species that, like $\mathrm{C} \mathrm{I}$, is not expected to be abundant in the shielded interiors of dense molecular clouds which have reached chemical equilibrium (e.g., Prasad and Huntress 1980). In fact its abundance is thought to be closely linked to the $\mathrm{C}$ I abundance. In a recent observational study of $\mathrm{C}_{2} \mathrm{H}$, Huggins, Carlson, and Kinney (1984; see also Wootten et al. 1980) have found that the ratio of $\mathrm{C}_{2} \mathrm{H}$ to ${ }^{13} \mathrm{CO}$ is 0.01 within a factor of about 2 for a wide variety of molecular clouds including M17 and S140. This is much higher than had been predicted from chemical models. Also the ratio of $\mathrm{C}_{2} \mathrm{H}$ to ${ }^{13} \mathrm{CO}$ does not vary over maps of objects, even over the edges of bright rims. Moreover the kinematics of the $\mathrm{C}_{2} \mathrm{H}$ is very similar to that of ${ }^{13} \mathrm{CO}$ and higher dipole moment molecules implying that they are spatially coexistent and that the $\mathrm{C}_{2} \mathrm{H}$ emission is not merely a halo around molecular clouds.

From the results presented here and the earlier observations of Phillips and Huggins (1981), our overall impression is that most of the $\mathrm{C}$ I emission seen toward dense clouds comes from the bulk of the clouds, not from the edges. However, it seems as though $\mathrm{C}_{\mathrm{I}}$ avoids the densest core regions. With the exception of the core regions, these results are similar to those found by Huggins, Carlson, and Kinney (1984) for the $\mathrm{C}_{2} \mathrm{H}$ molecule.

\section{SUMMARY}

From our observations of the edge-on $\mathrm{H}$ II regions M17 and S140 we find that the large observed $C$ I abundance cannot be explained by production within a few visual magnitudes of a cloud edge by an enhanced radiation field. The $C_{I}$ is widely spread throughout the clouds, and the column density appears to peak farther into the clouds than the $\mathrm{CO}$ column density. The derived $\mathrm{C} \mathrm{I} / \mathrm{CO}$ ratio is about $0.1-0.2$ inside these clouds.

We are grateful to M. Frerking, C. Masson, and E. Sutton for help with the observations. We also thank R. E. Miller of AT\&T Bell Labs for providing the SIS junctions used in the receivers of the OVRO $10 \mathrm{~m}$ telescopes for the CO observations. We acknowledge helpful discussions with N. Evans, M. Frerking, A. Glassgold, D. Hollenbach, W. Langer, and D. Williams. We thank the crew of the NASA Kuiper Airborne Observatory for their effective support. This work was supported by NASA grant NAG 2-1.

\section{APPENDIX}

\section{DERIVATION OF COLUMN DENSITIES}

For the analysis of the carbon chemistry of dense clouds, it is important to determine the abundance of free neutral carbon and its ratio to the abundance of carbon monoxide. We estimate CO column densities below using the standard LTE method (e.g., Dickman 1978; see also Spitzer 1978). This method assumes that the observed cloud is an infinite isothermal slab. The ${ }^{12} \mathrm{CO}$ line is optically thick, and we assume that the excitation temperature, $T_{x}$, is equal to the kinetic temperature of the gas. We also assume that the $\mathrm{C} \mathrm{I}$ and ${ }^{13} \mathrm{CO}$ excitation temperature is equal to that of ${ }^{12} \mathrm{CO}$. For the sake of clarity and because of the relatively high frequencies and temperatures involved in this study, we neglect any contribution to the flux from the cosmic background radiation throughout the following derivations. (The background was included in the actual calculations presented in Tables 2 and 4 ; it makes a difference of at most $15 \%$ for ${ }^{13} \mathrm{CO}$ and no difference for $\mathrm{C} \mathrm{I}$.)

In millimeter wave molecular line astronomy $T_{A}{ }^{*}$ is a unit which is defined to be a measure of intensity such that

$$
\frac{k T_{A}^{*}}{h v}=\frac{c^{2}}{2 h v^{3}} I_{v}
$$


and in the LTE case described above,

$$
\frac{k T_{A}^{*}}{h v}=\frac{c^{2}}{2 h v^{3}} B_{v}\left(T_{x}\right)\left[1-\exp \left(-\tau_{v}\right)\right]
$$

For $\tau \gg 1$, such as for ${ }^{12} \mathrm{CO}$,

$$
\frac{k T_{A}^{*}}{h v}=\left[\exp \left(\frac{h v}{k T_{x}}\right)-1\right]^{-1}
$$

which can be solved for $T_{x}$. At the peak of a less optically thick line, such as that of $\mathrm{C}$ I or ${ }^{13} \mathrm{CO}$,

$$
\exp \left(-\tau_{p}\right)=1-\frac{k T_{A}^{*}}{h v}\left[\exp \left(\frac{h v}{k T_{x}}\right)-1\right] \text {. }
$$

An alternative approach to the measurement of $\tau_{p}$ would be to use the curve of growth for line widths (Phillips et al. 1979; Phillips and Huggins 1981). The disadvantage of the latter method is that it requires observations of several isotopic species and transitions with different optical depths to construct the curve of growth.

The term $\tau_{p}$ is a function of the number of atoms or molecules in the lower state of the transition being considered. In both $\mathrm{C}$ I and $\mathrm{CO}$ we are dealing with ground-state transitions, i.e., ${ }^{3} P_{1} \rightarrow{ }^{3} P_{0}$ for $\mathrm{C}$ I and $J=1 \rightarrow 0$ for CO. So

$$
\tau_{v}=N_{0} S_{v}=N_{0} S \varphi(\Delta v)
$$

where $N_{0}$ is the number of $\mathrm{C}$ I atoms or $\mathrm{CO}$ molecules in the ground state and $\varphi(\Delta v)$ is the line profile, which we will assume is due to a Maxwellian velocity distribution. $S$ is the line strength; for atoms it is commonly written in terms of Einstein coefficients:

$$
S=\frac{c^{2}}{8 \pi v^{2}} \frac{g_{1}}{g_{0}} A_{10}\left[1-\exp \left(-\frac{h v}{k T_{x}}\right)\right],
$$

where $g_{0}(=1)$ and $g_{1}(=3)$ are the statistical weight of the ${ }^{3} P_{0}$ and ${ }^{3} P_{1}$ levels, respectively, and $A_{10}\left(=7.9 \times 10^{-8} \mathrm{~s}^{-1} ; \mathrm{Nussbau}-\right.$ mer 1971) is the Einstein coefficient for spontaneous emission. For the ground-state transition of a molecule

$$
S=\frac{8 \pi^{3} v}{3 h c}\left|\mu_{01}\right|^{2}\left[1-\exp \left(-\frac{h v}{k T_{x}}\right)\right],
$$

where $\left|\mu_{01}\right|^{2}=\mu^{2}$ (for $\mathrm{CO}, \mu=0.112$ debye). For a Maxwellian velocity distribution

$$
\varphi(0)=\frac{c}{v} \frac{1}{\Delta V_{i}}\left(\frac{4 \ln 2}{\pi}\right)^{1 / 2},
$$

where $\Delta V_{i}$ is the intrinsic FWHM of the velocity distribution, i.e., the width the line would have in the limit of zero optical depth. The factor $[(4 \ln 2) / \pi]^{1 / 2}$ equals 0.94 and hereafter will be dropped from our calculations. We must now account for finite optical depth in the lines. We find that the observed FWHM of the line, $\Delta V_{1 / 2}$, including saturation effects, is related to the intrinsic FWHM by

$$
\Delta V_{1 / 2}=\Delta V_{i} F\left(\tau_{p}\right)
$$

where

$$
\left.F\left(\tau_{p}\right)=\frac{1}{(\ln 2)^{1 / 2}}\left(\ln \llbracket \frac{\tau_{p}}{\ln \left\{2 /\left[\exp \left(-\tau_{p}\right)+1\right]\right\}}\right]\right)^{1 / 2}
$$

(Phillips et al. 1979). For small optical depths, $F\left(\tau_{p}\right) \approx 1$. For the largest optical depth considered here $F\left(\tau_{p}\right)=1.25$.

$$
\text { a) } \mathrm{C} \mathrm{I}
$$

For $\mathrm{C}$ I we have

$$
N\left(\mathrm{C}_{\mathrm{I}}\right)=N_{0}\left(\mathrm{C}_{\mathrm{I}}\right) \frac{f}{g_{0}},
$$

where $f$ is the partition function. In molecular clouds, which have fairly low temperatures, $\mathrm{C}$ I can be considered a three-level atom because none of the states above the ${ }^{3} P$ states are populated. Thus the partition function can be expressed simply as

$$
f=1+3 \exp \left(-h v / k T_{x}\right)+5 \exp \left(-E_{2} / k T_{x}\right),
$$

where $E_{2}$ is the energy of the ${ }^{3} P_{2}$ state above the ground state, $E_{2} / k=62.5 \mathrm{~K}$. On collecting terms

$$
N\left(\mathrm{C}_{\mathrm{I}}\right)=\tau_{p} \Delta V_{1 / 2} f \frac{8 \pi v^{3}}{3 c^{3} A_{10}}\left[1-\exp \left(-\frac{h v}{k T_{x}}\right)\right]^{-1} F\left(\tau_{p}\right)^{-1} .
$$

For $\tau_{p} \ll 1$ this equation reduces to Eqn. 3 of Phillips and Huggins (1981). 


\section{b) $\mathrm{CO}$}

The formulae for deriving the column density of ${ }^{13} \mathrm{CO}$ have been presented many times (see, e.g., Dickman 1978). In direct analogy with the above arguments for $\mathrm{C} \mathrm{I}$, but using as an estimate for the partition function

$$
f=\frac{2 k T_{x}}{h v},
$$

the column density of ${ }^{13} \mathrm{CO}$ can be expressed as

$$
N\left({ }^{13} \mathrm{CO}\right)=\tau_{p} \Delta V_{1 / 2} f \frac{3 h}{8 \pi^{3} \mu^{2}}\left[1-\exp \left(-\frac{h v}{k T_{x}}\right)\right]^{-1} F\left(\tau_{p}\right)^{-1} .
$$

This agrees with the formula of Dickman (1978) to within the factor $F\left(\tau_{p}\right)$.

\section{REFERENCES}

Bally, J., and Langer, W. D. 1982, Ap. J., 255, 143.

Beetz, M., Elsässer, H., Poulakos, C., and Weinberger, R. 1976, Astr. Ap., 50, 41.

Beichman, C. A., Becklin, E. E., and Wynn-Williams C. G. 1979, Ap. J. (Letters), 232, L47.

Beichman, C. A., Phillips, T. G., Wootten, H. A., and Frerking, M. A. 1982, in Regions of Recent Star Formation, ed. R. S. Roger and P. E. Dewdney (Dordrecht: Reidel), p. 445.

Blair, G. N., Evans, N. J., Vanden Bout, P. A., and Peters, W. L. 1978, Ap. J., 219, 896.

Boland, W., and de Jong, T. 1982, Ap. J., 268, 110. 1984, Astr. Ap., 134, 87.

Bradley, P. T., and Morton, D. C. 1969, Ap. J., 156, 687.

Chernoff, D. F., Hollenbach, D. J., and McKee, C. F. 1982, Ap. J. (Letters), 259, L97.

Chini, R., Elsässer, H., and Neckel, Th. 1980, Astr. Ap., 91, 186.

Chu, Y.-H., and Watson, W. D. 1983, Ap. J., 267, 151.

Clavel, J., Viala, Y. P., and Bel, N. 1978, Astr. Ap., 65, 435.

Crampton, D., and Fisher, W. A. 1974, Pub. Dom. Ap. Obs., 14, No. 12

Crampton, D., Georgelin, Y. M., and Georgelin, Y. P. 1978, Astr. Ap., 66, 1.

de Jong, T., Dalgarno, A., and Boland, W. 1980, Astr. Ap., 91, 68.

Dickman, R. L. 1978, Ap. J. Suppl., 37, 407.

Draine, B. T., and Roberge, W. G. 1982, Ap. J.(Letters), 259, L91.

Elmegreen, B. G., and Lada, C. J. 1976, A.J., 81, 1089.

Elmegreen, B. G., Lada, C. J., and Dickinson, D. F. 1979, Ap. J., 230, 415.

Falgarone, E., and Gilmore, W. 1981, Astr. Ap., 95, 32.

Felli, M., Churchwell, E., and Massi, M. 1984, Astr. Ap., 136, 53.

Felli, M., Johnston, K. J., and Churchwell, E. 1980, Ap. J. (Letters), 242, L157.

Frerking, M. A., Keene, J., Blake, G. A., Phillips, T. G., and Beichman, C. A. 1985, Ap. J., submitted.

Frerking, M. A., Langer, W. D., and Wilson, R. W. 1982, Ap. J., 262, 590.

Gatley, I., Becklin, E. E., Sellgren, K., and Werner, M. W. 1979, Ap. J., 233, 575.

Gerola, H., and Glassgold, A. E. 1978, Ap. J. Suppl., 37, 1

Glassgold, A. E., Huggins, P. J., and Langer, W. D. 1985, Ap. J., 290, 615.

Graedel, T. E., Langer, W. D., and Frerking, M. A. 1982, Ap. J.Suppl., 48, 321

Gull, T. R., and Balick, B. 1974, Ap. J., 192,63.

Harper, D. A., Low, F. J., Rieke, G. H., and Thronson, H. A. 1976, Ap. J., 205, 136.

Harvey, P. M., Campbell, M. F., and Hoffman, W. F. 1978, Ap. J., 219, 891.

Hayashi, M., Omodaka, T., Hasegawa, T., and Suzuki, S. 1985, Ap. J., 288, 170.

Herbst, E. 1983, Ap.J.S Suppl., 53,41.

Hickok, F. R., and Morton, D. C. 1968, Ap. J., 152, 203

Hildebrand, R. H., Loewenstein, R. F., Harper, D. A., Orton, G. S., Keene, J., and Whitcomb, S. E. 1985, Icarus, in press.

Huggins, P. J., Carlson, W. J., and Kinney, A. L. 1984, Astr. Ap., 133, 347.

Icke, V., Gatley, I., and Israel, F. P. 1980, Ap. J., 236, 808.

Iglesias, E. 1977, Ap. J., 218, 697.

Jaffe, D. T. 1980, Ph.D. thesis, Harvard University.

Kleinmann, D. E., and Wright, E. L., 1973, Ap. J.(Letters), 185, L131.

Knapp, G. R., Brown, R. L., Kuiper, T. B. H., and Kakar, R. K. 1976, Ap. J., 204, 781 .
Krolik, J. H., and Kallman, T. R. 1983, Ap. J., 267, 610

Kutner, M. L., and Ulich, B. L. 1981, Ap. J., 250, 341.

Lada, C. J. 1976, Ap. J. Suppl., 32, 603.

Langer, W. $1976 a, A p . J .$, 206, 699.

- 1976b, Ap. J., 210, 328.

Langer, W. D., Graedel, T. E., Frerking, M. A., and Armentrout, P. B. 1984, Ap. J., 277, 581 .

Leung, C. M., Herbst, E., and Huebner, W. F. 1984, Ap. J. Suppl., 56, 231.

Linke, R. A., and Goldsmith, P. F. 1980, Ap. J., 235, 437

Linsky, J. L. 1973, Ap. J. Suppl., 25, 163.

Liszt, H. S., and Linke, R. A. 1975, Ap. J., 196, 709.

Masson, C. R. 1982, Astr. Ap., 114, 270.

Montemerle, T., Koch-Miramond, L., Falgarone, E., and Grindlay, J. E. 1983, Ap. J., 269, 182

Mundy, L. G. 1984, Ph.D. thesis, University of Texas.

Norman, C., and Silk, J. 1980, Ap. J., 238, 158.

Nussbaumer, H. 1971, Ap.J., 166, 411 .

Panagia, N. 1973, A.J., 78, 929.

Phillips, T. G., and Huggins, P. J. 1981, Ap. J., 251, 533.

Phillips, T. G., Huggins, P. J., Wannier, P. G., and Scoville, N. Z. 1979, Ap. J., 231, 720 .

Phillips, T. G., and Jefferts, K. B. 1974, IEEE Trans., MTT-22, 1290.

Phillips, T. G., Jefferts, K. B., and Wannier, P. G. 1973, Ap. J. (Letters), 186 L19.

Prasad, S. S., and Huntress, W. T. 1980, Ap. J., 239, 151.

1982, Ap. J., 260, 590.

Prasad, S. S., and Tarafdar, S. P. 1983, Ap. J., 267, 603.

Saykally, R. J., and Evenson, K. M. 1980, Ap. J. (Letters), 238, L107.

Schwartz, P. R., Thronson, H. A., Lada, C. J., Smith, H. A., Glaccum, W., Harper, D. A., and Knowles, S. H. 1983, Ap. J., 271, 625.

Smith, D., and Adams, N. G. 1984, Ap. J. (Letters), 284, L13.

Snell, R. L., Mundy, L. G., Goldsmith, P. F., Evans, N. J., and Erickson, N. R. 1984, Ap. J., 276, 625.

Spitzer, L. 1978, Physical Processes in the Interstellar Medium (New York: Wiley), § 3.2 .

Tarafdar, S. P., Prasad, S. S., and Hunteress, W. T. 1983, Ap. J., 267, 156.

Tarafdar, S. P., Prasad, S. S., Huntress, W. T., Villere, K. R., and Black, D. C. 1985, Ap. J., 289, 220.

Thronson, H. A., and Lada, C. J. 1983, Ap. J., 269, 175.

Tielens, A. G. G. M., and Hollenbach, D. 1985a, Icarus, 61, 40.

$\longrightarrow .1985 b, A p . J .291,722$.

Tokunaga, A. T., and Thompson, R. I. 1979, Ap. J, 229, 583

Williams, D. A., and Hartquist, T. W. 1984, M.N.R.A.S., $210,141$.

Wilson, T. L., Fazio, G. G., Jaffe, D., Kleinmann, D., Wright, E. L., and Low, F. J. 1979, Astr. Ap., 76, 86.

Wootten, A., Bozyan, E. P., Garrett, D. B., Loren, R. B., and Snell, R. L. 1980, Ap. J., 239, 844

Wootten, A., Phillips, T. G., Beichman, C. A., and Frerking, M. A. 1982, Ap. J., (Letters), 256, L5.

C. A. Beichman: Jet Propulsion Laboratory, 230-207, Pasadena, CA 91109

Geoffrey A. Blake, Jocelyn Keene, and T. G. Phillips: California Institute of Technology, 320-47, Pasadena, CA 91125

Patrick J. Huggins: Physics Department, New York University, 4 Washington Place, New York, NY 10003 